\title{
Problem with your pump? Just swap it out
}

\author{
Nahush A. Mokadam, MD, FACC, FACS
}

See related article on pages $267-78$.

Worldwide, the use of left ventricular assist devices (VADs) continues to increase dramatically, both as bridge to transplant and as destination therapy. The issue of VAD thrombosis is currently clinically very relevant, as there has been an observation that this phenomenon may be increasing. ${ }^{1,2}$ One of the most important treatments for this potentially fatal situation is device exchange (Figure 1). In the literature, 2-year survival after exchange is reported to be decreased relative to primary implantation, from $69 \%$ to $56 \%{ }^{2}$

In the most recent report from the Interagency Registry for Mechanically Assisted Circulatory Support (INTERMACS), freedom from pump exchange has decreased from $96 \%$ in the era encompassing 2008 through 2010 to $91 \%$ in the era encompassing 2011 through $2013 .{ }^{3}$ This report accounted for 795 events overall, and 522 exchanges in the most recent era. The cause of this change remains under much scrutiny, with speculation ranging from pump design changes to differences in patient profiles, to an increasing use of left VADs as destination therapy. The challenges for the mechanical circulatory support (MCS) community lie in determining how best to predict which patients are at risk for these events, promptly diagnosing and treating device malfunction, and responsibly stewarding this expensive technology.

In this issue of the Journal of Thoracic and Cardiovascular Surgery, Anand and colleagues ${ }^{4}$ report a single-center retrospective experience with continuous-flow VAD exchange. This series represents the largest of its type and provides the MCS community with an excellent benchmark. The report focuses on 77 left VAD exchanges in 66 patients during the course of the last 14 years. Most of the exchanges were HeartMate II (Thoratec Corporation, Pleasanton, Calif) devices $(\mathrm{n}=59)$; also represented were Jarvik 2000 (Jarvik Heart, Inc, New York, NY) devices $(\mathrm{n}=10)$ and HeartWare HVAD (HeartWare Inc, Framingham,

\footnotetext{
From the Division of Cardiothoracic Surgery, University of Washington Medical Center, Seattle, Wash.

Disclosures: Nahush A. Mokadam reports consulting fees from Thoratec, Syncardia, and HeartWare.

Received for publication Aug 12, 2014; accepted for publication Aug 13, 2014; available ahead of print Sept 11, 2014.

Address for reprints: Nahush A. Mokadam, MD, FACC, FACS, Division of Cardiothoracic Surgery, University of Washington Medical Center, 1959 NE Pacific St, Box 356310, Seattle, WA 98195 (E-mail: mokadamn@uw.edu).

J Thorac Cardiovasc Surg 2015;149:279-80

$0022-5223 / \$ 36.00$

Copyright (C) 2015 by The American Association for Thoracic Surgery http://dx.doi.org/10.1016/j.jtcvs.2014.08.024
}

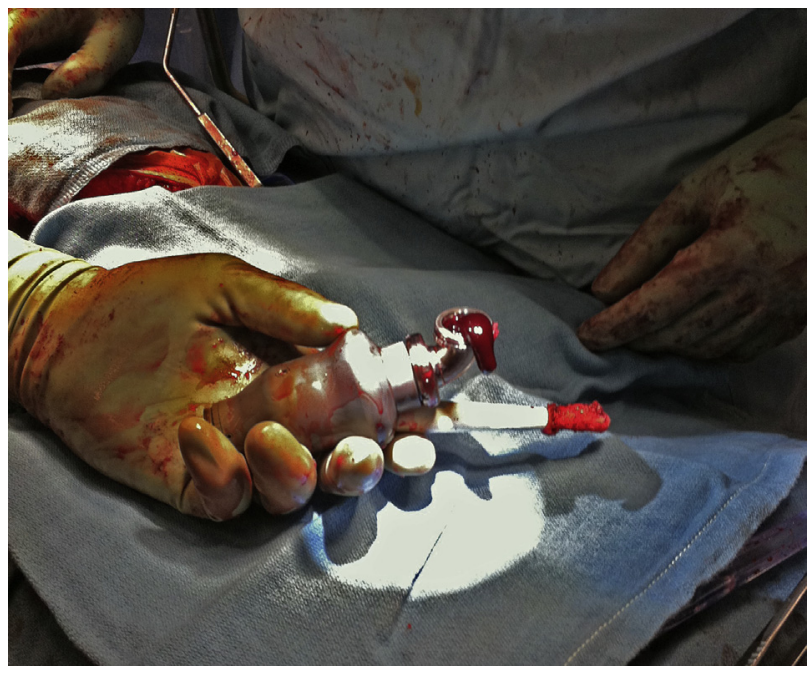

FIGURE 1. Left ventricular assist device exchange for pump thrombosis.

Mass) devices $(n=15)$, thus encompassing the most commonly implanted devices. The main surprising finding was that overall survival from initial implantation was not different between patients who required exchange and those who did not. At 4-year follow-up, both groups had a survival greater than $50 \%$. In fact, in this analysis, pump exchange was an independent positive predictor of survival $(P=$ .005). This is remarkable; most practitioners have assumed pump exchange to be a poor prognostic marker for survival because of the higher incidence of infection, stroke, and gastrointestinal bleeding seen after an exchange. ${ }^{2}$

In an attempt to determine an etiology for the increasing pump exchanges, Anand and colleagues ${ }^{4}$ performed multivariate analysis of preoperative characteristics. Surprisingly, a preoperative neurologic event and the need for preoperative temporary MCS were the only predictors of the need for pump exchange. The relationship between preoperative neurologic events and pump exchange is not entirely clear and warrants further investigation. As Anand and colleagues ${ }^{4}$ suggest, it may be reasonable to include a functionality assessment and hypercoagulable workup in the assessment of these patients. At the same time, this provides the MCS community with another factor to consider in patients undergoing primary VAD implantation. Clearly we as a community have a responsibility to remain focused on factors that may precipitate pump dysfunction.

Finally, this report provokes an ethical discussion. When is it reasonable to withhold a pump exchange, if there is no difference in overall survival? This is particularly germane to patients who undergo VAD implantation as destination 
therapy and who do not have a feasible transplant exit strategy. Other than for multiorgan dysfunction and medical noncompliance, can we reasonably deny patients access to a second pump? A third pump? The debate will certainly continue.

The field of MCS continues to evolve with rapidly changing technology, miniaturization, improving biocompatibility, and our collective clinical experience. The proof of concept of MCS to support a failing heart has been long established. We now have evolving evidence that more than one pump can reliably prolong life. We will soon enter an era of smaller devices, potentially more forgiving on the coagulation cascade, and even fully implantable systems. Eventually, we may reach a time when we have a permanent mechanical replacement for the failing heart. Until that time, if you have a problem with your pump, do you just swap it out?

\section{References}

1. Starling RC, Moazami N, Silvestry SC, Ewald G, Rogers JG, Milano CA, et al. Unexpected abrupt increase in left ventricular assist device thrombosis. $N$ Engl J Med. 2014;370:33-40.

2. Kirklin JK, Naftel DC, Kormos RL, Pagani FD, Myers SL, Stevenson LW, et al. Interagency Registry for Mechanically Assisted Circulatory Support (INTERMACS) analysis of pump thrombosis in the HeartMate II left ventricular assist device. J Heart Lung Transplant. 2014;33:12-22.

3. Kirklin JK, Naftel DC, Pagani FD, Kormos RL, Stevenson LW, Blume ED, et al. Sixth INTERMACS annual report: a 10,000-patient database. J Heart Lung Transplant. 2014;33:555-64.

4. Anand J, Singh SK, Hernandez R, et al. Continuous-flow ventricular assist device exchange is safe and effective in prolonging support time in patients with endstage heart failure. J Thorac Cardiovasc Surg. 2015;149:267-78. 